\title{
Experimental Highlights: Heavy Quark Physics in Heavy-Ion Collisions at RHIC
}

\author{
Rachid Nouicer ${ }^{1, a}$ \\ ${ }^{1}$ Physics Department, Brookhaven National Laboratory, Upton, New York 11973, United States
}

\begin{abstract}
The discovery at RHIC of large high- $p_{\mathrm{T}}$ suppression and flow of electrons from heavy quarks flavors have altered our view of the hot and dense matter formed in central $\mathrm{Au}+\mathrm{Au}$ collisions at $\sqrt{s_{N N}}=200 \mathrm{GeV}$. These results suggest a large energy loss and flow of heavy quarks in the hot, dense matter. In recent years, the RHIC experiments upgraded the detectors; (1) PHENIX Collaboration installed silicon vertex tracker (VTX) at midrapidity region and forward silicon vertex tracker (FVTX) at the forward rapidity region, and (2) STAR Collaboration installed the heavy flavor tracker (HFT) and the muon telescope detector (MTD) both at the mid-rapidity region. The PHENIX experiments established measurements of $\psi(1 S)$ and $\psi(2 S)$ production as a function of system size, $p+p$, $p+\mathrm{Al}, p+\mathrm{Au}$, and ${ }^{3} \mathrm{He}+\mathrm{Au}$ collisions at $\sqrt{s_{N N}}=200 \mathrm{GeV}$. In $\mathrm{p} /{ }^{\beta} \mathrm{He}+\mathrm{A}$ collisions at forward rapidity, we observe no difference in the $\psi(2 S) / \psi(1 S)$ ratio relative to $p+p$ collisions. At backward rapidity, where the comoving particle density is higher, we find that the $\psi(2 S)$ is preferentially suppressed by a factor of two. STAR Collaboration presents the first $J / \psi$ measurements in the di-muon decay channel in $\mathrm{Au}+\mathrm{Au}$ at $\sqrt{s_{N N}}=200 \mathrm{GeV}$ at mid-rapidity. We observe a clear $J / \psi R_{\mathrm{AA}}$ suppression and qualitatively well described by transport models, including dissociation and regeneration simultaneously.
\end{abstract}

\section{Physics Motivation}

Hadrons conveying heavy quarks, i.e. charm $(c)$ or bottom $(b)$, are consider important probes of the hot and dense medium, Quark Gluon Plasma (QGP), created in relativistic heavy-ion collisions. Heavy quark-antiquark pairs are mainly produced in initial hard scattering processes of partons. While some of the produced pairs form bound quarkonia, the vast majority hadronize into open heavy flavor particles. They interact through the medium and are expected to be sensitive to its energy density through the mechanism of parton energy loss. Due to the large mass of heavy quarks the suppression of small-angle gluon radiation should reduce their energy loss, and consequently any suppression of heavy-quark mesons like $D$ and $B$ mesons at high- $p_{T}$ is expected to be smaller than that observed for hadrons consisting of light quarks [1].

The evolution of the nuclear modification factor for charged hadrons and $\pi^{0}$ with center-of-mass energy, from the SPS to RHIC and then to the LHC is presented in figure 1 . We observe that in the presented $p_{\mathrm{T}}$ region, charged hadron production at the LHC is found to be about $50 \%$ more suppressed than at RHIC, and has a similar suppression value as for neutral pions $\left(\pi^{0}\right)$ measured by PHENIX.

\footnotetext{
ae-mail: rachid.nouicer@bnl.gov
} 


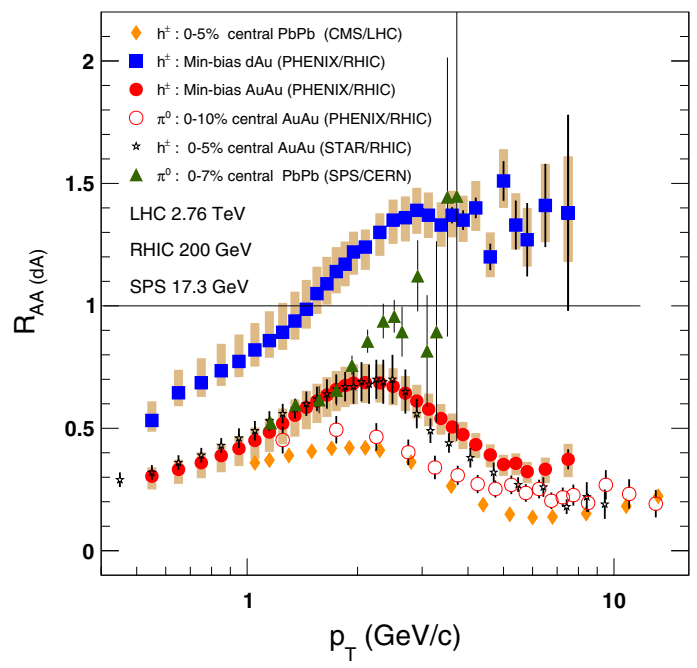

Figure 1. The evolution of the nuclear modification factors, $R_{\mathrm{AA}}$, with center-of-mass energy, from the SPS to RHIC and then to the LHC. The error bars correspond to the statistical errors. For clarity, the systematic errors are shown as vertical bands. For more details see Ref. [2].

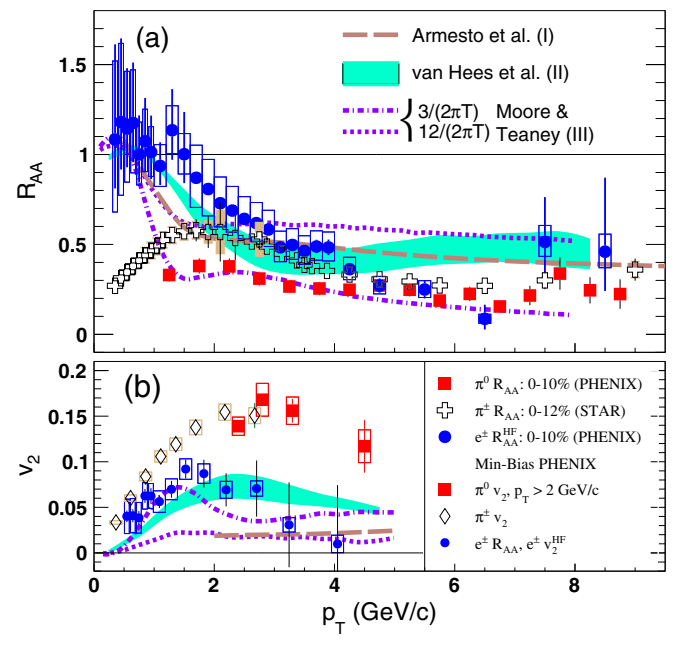

Figure 2. The nuclear modification factor, $\mathrm{R}_{\mathrm{AA}}^{\mathrm{HF}}$, for HF electrons compared with the $\mathrm{R}_{\mathrm{AA}}$ of $\pi^{0}$ in central $\mathrm{Au}+\mathrm{Au}$ collisions at $\sqrt{s_{N N}}=200 \mathrm{GeV}$, see panel (a). Panel (b) considers the anisotropic flow of HF electrons $\mathrm{v}_{2}^{H F}$ with that of $\mathrm{v}_{2}$ of $\pi^{0}$ and $\pi^{ \pm}$in minimumbias $\mathrm{Au}+\mathrm{Au}$ collisions. For more details see Ref. [2]

These measurements of $R_{A A}\left(p_{\mathrm{T}}\right)$ at RHIC and LHC is consistent with a large energy loss in the medium causing it to become opaque to the propagation of high momentum partons [2,3]. In contrast, $R_{A A}\left(p_{\mathrm{T}}\right)$ for $\pi^{0}$ at SPS energy exhibit enhancement, as shown in figure 1. This effect of $R_{A A}\left(p_{\mathrm{T}}\right)$ suppression of the high- $p_{\mathrm{T}}$ hadron spectra at the mid-rapidity region in central $\mathrm{A}+\mathrm{A}$ collisions, proposed by Bjorken, Gyulassy, and others $[4,5]$ rests on the expectation of a large energy loss of high- $p_{\mathrm{T}}$ partons scattered in the initial stages of collisions in a medium with a high density of free color charges.

Adding to this discovery of suppression of particles at high- $p_{\mathrm{T}}$, two very striking results were seen for open heavy flavor from the PHENIX experiment via the measurement of electrons $\left(e_{H F}^{ \pm}\right)$from the semi-leptonic decays of hadrons carrying charm or bottom quarks. First, heavy mesons, despite their large mass, exhibit a suppression at high transverse momentum compared to that expected from $p+p$ collisions $[2,6-8]$. This suppression is similar to that of light mesons, which implies a substantial energy loss of fast heavy quarks while traversing the medium, see figure 2(a). Second, an elliptic flow is observed for heavy mesons, electrons from semi-leptonic decays of hadrons carrying charm or bottom quarks, that is comparable to that of light mesons like pions, as shown in figure 2(b). This implies that the heavy quarks in fact are sensitive to the pressure gradients driving hydrodynamic flow giving new insights into the strongly coupled nature of the QGP fluid at these temperatures.

\section{Latest Heavy Flavor Results from the PHENIX Experiment at RHIC}

In recent years, the PHENIX Collaboration has installed a silicon vertex tracker both in central rapidity (VTX) and in forward rapidity (FVTX) regions, and has collected large data samples [9, 10]. These two silicon trackers enhance the capability of heavy flavor measurements via precision tracking. 


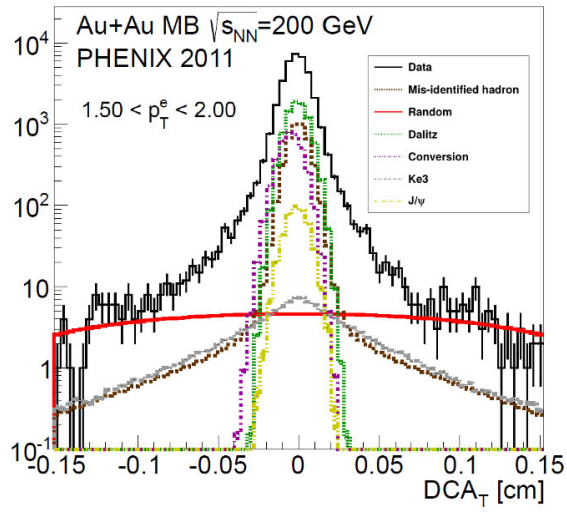

Figure 3. The $\mathrm{DCA}_{T}$ distribution for electrons with $1.5<p_{\mathrm{T}}^{e}<2.0 \mathrm{GeV} / \mathrm{c}$ in $\mathrm{Au}+\mathrm{Au}$ minimum-bias collisions at $\sqrt{s_{N N}}=200 \mathrm{GeV}$. Also shown are the normalized contributions for the various background components [18].

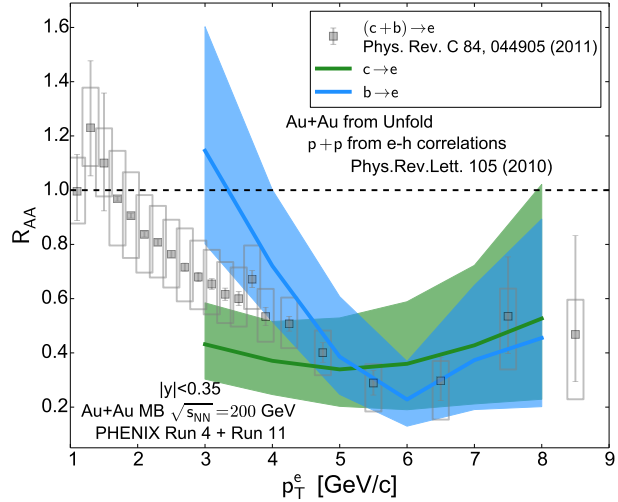

Figure 4. The nuclear modification factors $R_{\mathrm{AA}}$ of electrons from charm $(c \rightarrow e)$, bottom $(b \rightarrow e)$ decays and combined heavy flavor as a function of $p_{\mathrm{T}}^{e}$ in $\mathrm{Au}+\mathrm{Au}$ minimum-bias collisions at $\sqrt{s_{N N}}=200 \mathrm{GeV}$ [18].

\subsection{Open Heavy Flavor}

Open heavy flavor production is measured in PHENIX experiment through the measurement of inclusive electrons or muons [11-14]. These analyses use a cocktail method to remove fake and real electrons/muons from the data sample. i.e. for the electron measurement, the electrons that come from either meson decay or photon conversions are measured and subtracted from the inclusive spectrum and the remainder is attributed to electrons $\left(e_{H F}^{ \pm}\right)$coming from the semi-leptonic decay of $D$ and $B$ mesons. The large suppression $R_{\mathrm{AA}}$ of $e_{H F}^{ \pm}$at high- $p_{\mathrm{T}}$ in $\mathrm{Au}+\mathrm{Au}$ collisions at $\sqrt{s_{N N}}=200 \mathrm{GeV}$, shown in figures 2 , and similar to that of light mesons $\pi^{0}$, is the more striking because perturbative QCD calculations indicate a substantial contribution from bottom quark decays for $p_{\mathrm{T}}>5 \mathrm{GeV} / \mathrm{c}[15]$. For the specific purpose of separating the contributions of charm and bottom quarks at mid-rapidity, the PHENIX Collaboration has added micro-vertexing capabilities in the form of a silicon vertex tracker (VTX) $[9,10]$. The different lifetimes and kinematics for charm and bottom hadrons decaying to electrons enable separation of their contributions with measurements of displaced tracks [18, 19].

Figure 3 shows the $\mathrm{DCA}_{T}$ distribution (in transverse plane) for electrons $\left(e^{ \pm}\right)$in $1.5<p_{\mathrm{T}}^{e}<2.0 \mathrm{GeV} / \mathrm{c}$ range obtained in $\mathrm{Au}+\mathrm{Au}$ minimum-bias collisions at $\sqrt{s_{N N}}=200 \mathrm{GeV}$ using data set of RHIC Run 2011. In order to extract the heavy flavor contributions, all background components, as shown in figure 3 , were fully accounted for and their $\mathrm{DCA}_{\mathrm{T}}$ shapes as a function of $p_{\mathrm{T}}$ incorporated. The nuclear modifications, $R_{A A}^{c \rightarrow e}$, and $R_{A A}^{b \rightarrow e}$, for charm and bottom hadron decays respectively are presented in figure 4 and detailed in Ref [18]. We note that a significantly larger data set of $\mathrm{Au}+\mathrm{Au}$ collisions at $\sqrt{S_{N N}}=200 \mathrm{GeV}$ was collected in 2014 .

\subsection{Quarkonia Measurements - $\psi(1 S)$ and $\psi(2 S)$ Mesons Production Versus System Size}

One method to separate final-state effects is through studies of states with the same quark content but different binding energies, such as the charmonium states $\psi(1 S)$ and $\psi(2 S)[20,21]$. Although significant initial-state effects on open charm have been found in $d+\mathrm{Au}$ collisions at RHIC, these 

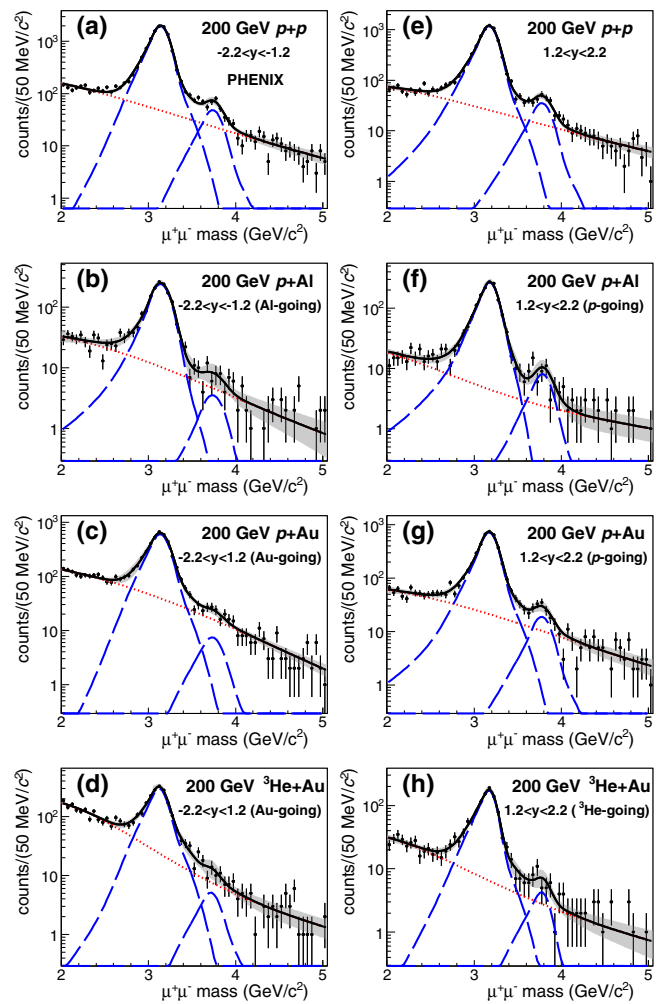

Figure 5. The measured di-muon mass spectra with fits from the (in the A-going direction: a-d) South and (in the $p$-going direction: $\mathrm{e}-\mathrm{h}$ ) North PHENIX muon arms, for $p+p, p+\mathrm{Al}, p+\mathrm{Au}$, and ${ }^{3} \mathrm{He}+\mathrm{Au}$ collisions at $\sqrt{s_{N N}}=200 \mathrm{GeV}$. The total fit is the solid [black] line with a shaded [gray] band representing the $90 \%$ confidence level. The dashed [blue] and dotted [red] lines represent the contributions from the resonances and background, respectively. For more details see Ref. [21].

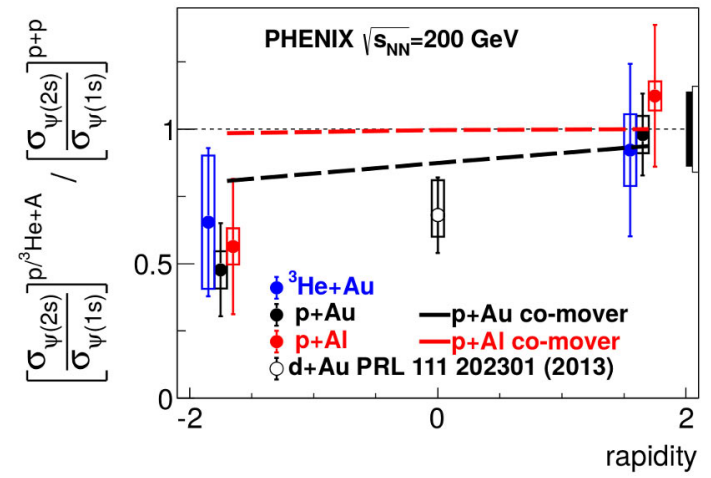

Figure 6. The double ratio of $\psi(2 S) / \psi(1 S)$ mesons measured in in $p+\mathrm{Al}, p+\mathrm{Au}$, and ${ }^{3} \mathrm{He}+\mathrm{Au}$ collisions to that same ratio in $p+p$ collisions at $\sqrt{s_{N N}}=200 \mathrm{GeV}$, with a calculation based on breakup by comoving particles. The bars (boxes) on the data points represent the statistical (systematic) uncertainties, and the shaded (open) box around unity represents the global uncertainty on the forward/backward (mid) rapidity data. For more details see Ref. [21].

should equally affect all charm pairs before projection onto a final state. Therefore any differences in the modification of $\psi(2 S)$ and $\psi(1 S)$ production are likely due to late time effects which are sensitive to differences in the fully-formed meson radius and binding energy [21].

Figure 5 shows the measured di-muon mass spectra from $p+p, p+\mathrm{Al}, p+\mathrm{Au}$, and ${ }^{3} \mathrm{He}+\mathrm{Au}$ collisions at $\sqrt{s_{N N}}=200 \mathrm{GeV}$. These distributions comprise peaks at the $\psi(1 S)$ and $\psi(2 S)$ masses (3.1 and $3.7 \mathrm{GeV} / c^{2}$, respectively) on top of correlated background from charm and bottom hadron decays and Drell-Yan pairs, plus combinatorial background from light meson decays and hadrons, which are not stopped in the absorbers [21]. From figure 5, it is apparent that the $\psi(2 S)$ peaks are suppressed relative to the $\psi(1 S)$ peak in the columns on the left (in the A-going direction). Quantitative comparisons are accomplished by calculating the double ratio of $\psi(2 S) / \psi(1 S)$ production in $\mathrm{p} / \mathrm{He}+\mathrm{A}$ 


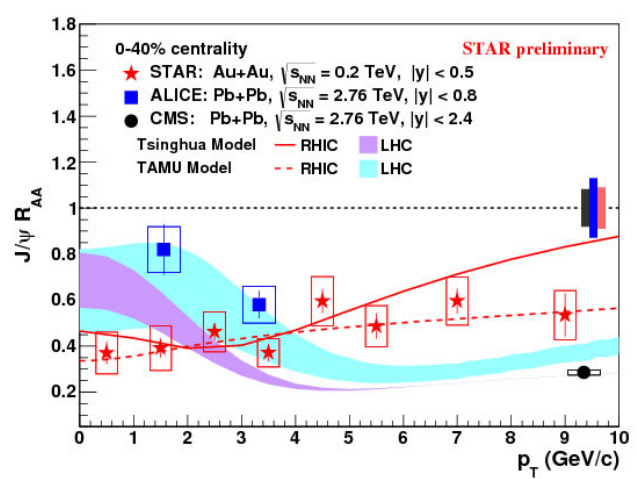

Figure 7. The nuclear modification factors $R_{\mathrm{AA}}$ as a function of $p_{\mathrm{T}}$ at RHIC (red star) and at LHC (blue square, black circle). The lines and bands indicate transport model calculations for RHIC and LHC energies [22].

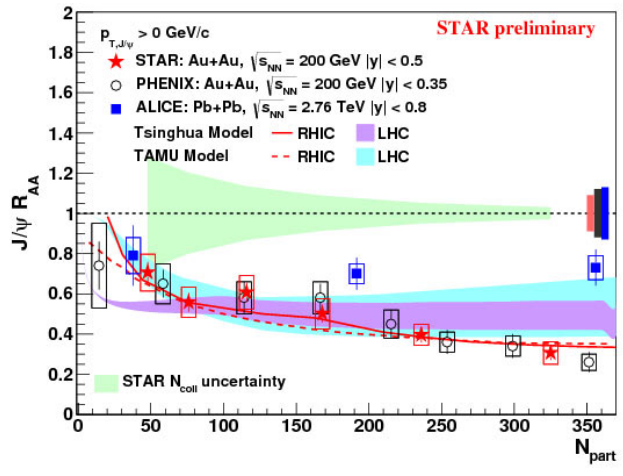

Figure 8. The nuclear modification factors $R_{\mathrm{AA}}$ for integrated $p_{\mathrm{T}}$ as a function of $\mathrm{N}_{\text {part }}$ in $\mathrm{Au}+\mathrm{Au}$ and $\mathrm{Pb}+\mathrm{Pb}$ collisions at RHIC and LHC energies respectively [22].

collisions to the ratio found in $p+p$ collisions, as shown in figure 6. At forward rapidity, the double ratio is consistent with unity in all three collision systems, indicating that any possible nuclear effects on the two charmonium states are comparable. Because these states are not fully formed until after they exit the nucleus, the fact that any nuclear effects have an equal magnitude on both states suggests that there are no significant final state effects on the pair which occur in this rapidity region. At backward rapidity, the ratios in all collision systems are suppressed by a factor of 2 . The mechanism for this preferential suppression of the $\psi(2 S)$ relative to the $\psi(1 S)$ is expected to occur after the $\psi(1 S)$ formation time. A significant difference in the late stages of the collision between backward rapidity and forward rapidity regions suggests the presence of a larger number of comoving hadrons [21].

\section{Latest Heavy Flavor Results from the STAR Experiment at RHIC}

The rare production of heavy quarks and large combinatorial background in heavy-ion collisions makes the measurements of heavy flavor particles very difficult with the STAR detectors. To overcome the challenge of the combinatorial background, the STAR Collaboration upgraded the detector by adding the heavy flavor tracker (HFT) and the muon telescope detector (MTD) in 2014.

\subsection{Measurements of $J / \psi$ in the Di-muon Decay Channel in Au + Au at Mid-rapidity}

Figure 7 shows the nuclear modification factor $R_{\mathrm{AA}}$ of $J / \psi$ measurements in the di-muon decay channel in $0-40 \%$ central $\mathrm{Au}+\mathrm{Au}$ collisions at $\sqrt{s_{N N}}=200 \mathrm{GeV}$ at mid-rapidity, compared with LHC results [22-24]. We observe a strong suppression at RHIC at low $p_{\mathrm{T}}$ indicating that dissociation plays a significant role in this $p_{\mathrm{T}}$ range. The increasing trend of $R_{\mathrm{AA}}$ at RHIC at high- $p_{\mathrm{T}}$ region could be interpreted by formation-time effects and feed-down of $B$ hadrons. The least suppression of $J / \psi$ at LHC at low- $p_{\mathrm{T}}$ indicates larger regeneration contribution due to higher charm cross section, while more suppression of $J / \psi$ at LHC at high- $p_{\mathrm{T}}$ indicates larger dissociation rate due to higher temperature of the medium [22]. Transport Models [25-28], including dissociation and regeneration effects, describe qualitatively the $R_{\mathrm{AA}}$ of $J / \psi$ as a function of $p_{\mathrm{T}}$ at RHIC and LHC given energies. Figure 8 
presents the $J / \psi R_{\mathrm{AA}}$ centrality dependence for integrated $p_{\mathrm{T}}$. We observe that both models reproduce centrality dependence at RHIC, but aim to overestimate suppression (or underestimate $R_{\mathrm{AA}}$ of $J / \psi$ ) at LHC.

\section{Conclusions}

STAR and PHENIX Measurements of heavy flavor made in heavy-ion collisions at RHIC have led to a broad and deep understanding of the properties of hot QCD matter. Recently, the PHENIX experiments established measurements of $\psi(1 S)$ and $\psi(2 S)$ production as a function of system size, $p+p$, $p+\mathrm{Al}, p+\mathrm{Au}$, and ${ }^{3} \mathrm{He}+\mathrm{Au}$ collisions at $\sqrt{s_{N N}}=200 \mathrm{GeV}$. In $\mathrm{p} /{ }^{3} \mathrm{He}+\mathrm{A}$ collisions at forward rapidity, we observe no difference in the $\psi(2 S) / \psi(1 S)$ ratio relative to $p+p$ collisions. At backward rapidity, where the comoving particle density is higher, we find that the $\psi(2 S)$ is preferentially suppressed by a factor of two. STAR Collaboration presents the first $J / \psi$ measurements in $\mathrm{Au}+\mathrm{Au}$ collisions at $\sqrt{s_{N N}}=200 \mathrm{GeV}$ in the di-muon decay channel at mid-rapidity region. We observe a clear $J / \psi R_{\mathrm{AA}}$ suppression and qualitatively well described by transport models.

\section{References}

[1] Y. L. Dokshitzer and D. E. Kharzeev, Phys. Lett. B 519, 199 (2001)

[2] R. Nouicer, European Physical Journal Plus 131, 70 (2016)

[3] S. Mehrabi Pari et al., International Journal of Modern Physics E, 25, No. 6, 1650040 (2016)

[4] J. D. Bjorken, Phys. Rev. D 27, 140 (1983)

[5] M. Gyulassy et al. Phys. Lett. B 243, 432 (1990)

[6] S.S. Adler et al. (PHENIX Collaboration), Phys. Rev. Lett. 98, 172302 (2007)

[7] S.S. Alder et al. (PHENIX Collaboration), Phys. Rev. Lett. 96, 032301 (2006)

[8] J. Adams et al. (STAR Collaboration), Phys. Rev. Lett. 94, 062301 (2005)

[9] R. Nouicer et al., Journal of Instrumentation (JINST) 4, P04011 (2009)

[10] R. Nouicer et al., Nuclear Instruments and Methods in Physics Research B 261, 10671071 (2007)

[11] R. Nouicer et al. (PHENIX Collaboration), Journal of Physics : Conf. Series 420, 012021 (2013)

[12] R. Nouicer et al. (PHENIX Collaboration), Nuclear Physics A 862, 64 (2011)

[13] A. Adare et al. (PHENIX Collaboration), Phys. Rev. Lett. 109, 242301 (2012)

[14] A. Adare et al. (PHENIX Collaboration), Phys. Rev. Lett. 112, 252301 (2014)

[15] M. Cacciari et al., Phys. Rev. Lett. 95, 122001 (2005)

[16] A. Adare et al. (PHENIX Collaboration), Phys. Rev. C 86, 024909 (2012)

[17] R. Sharma et al., Phys. Rev. C 80, 054902 (2009)

[18] A. Adare et al. (PHENIX Collaboration), Phys. Rev. Lett. 93, 034904 (2016)

[19] R. Nouicer et al. (PHENIX Collaboration), Nuclear Physics A 904-905C, 647 (2013)

[20] H. Satz, Journal of Physics G 32, R25 (2006)

[21] A. Adare et al. (PHENIX Collaboration), arXiv:1609.06550 [nucl-ex]

[22] T. Todoroki et al. (STAR Collaboration), arXiv:1612.02499

[23] B. Abelev et al. (ALICE Collaboration), Phys. Let. B 734, 314 (2014)

[24] S. Chatrchyan et al. (CMS Collaboration), JHEP 05, 063 (2012)

[25] Y. Liu et al., Phys. Let. B 678, 72 (2009)

[26] K. Zhou et al., Phys. Rev. C 89, 054911 (2014)

[27] X. Zhao et al., Phys. Rev. C 82, 064905 (2010)

[28] X. Zhao et al., Nucl. Phys. A 859, 114 (2011) 